\title{
XI. On achromatic interference-bands
}

\section{Lord Rayleigh Sec. R.S.}

To cite this article: Lord Rayleigh Sec. R.S. (1889) XI. On achromatic interference-bands , Philosophical Magazine Series 5, 28:171, 77-91, DOI: 10.1080/14786448908619834

To link to this article: http://dx.doi.org/10.1080/14786448908619834

$$
\text { 曲 Published online: } 08 \text { May } 2009 .
$$

Submit your article to this journal 준

LII Article views: 8

Q View related articles $\asymp$ 
LONDON, EDINBURGH, AND DUBLIN

\section{PHILOSOPHICAL MAGAZINE}

AND

\section{JOURNAL OF SCIENCE.}

[FIFTH SERIES.]

$A \overline{U G U S T 1889 .}$

XI. On Achromatic Interference-Bands. By Lord RAYLeIGH, Sec. R.S., Professor of Natural Philosophy in the Royal Institution*.

\section{Introduction.}

WHEN there is interference of light, the width of the resulting bands, measured for example from darkness to darkness, is usually a function of the colour of the light employed. Thus, in the case of Fresnel's well-known interference-experiment, in which light reflected from two slightly inclined mirrors illuminates a screen, the width of the bands is proportional to the wave-length of the light. In order that a considerable number of bands may be visible, it is necessary that the light be highly homogeneous ; otherwise it is impossible that the various band-systems can fit one another over the necessary range. If the light could be supposed to be absolutely homogeneous, there would be no limit to the number of observable bands : and, what is especially to be remarked, there would be nothing by which one band could be distinguished from another,-in particular there could be no central band recognizable. When, on the other hand, the light is white, there may be a central band at which all the maxima of brightness coincide ; and this band, being white, may be called the achromatic band. But the system of bands is not usually achromatic. Thus, in Fresnel's experiment the centre of symmetry fixes the position of the central achromatic band,

* Communicated by the Author.

Plil. Mag. S. 5. Vol. 28. No. 171. August 1889. H 
but the system is far from achromatic. Theoretically there is not even a single place of darkness, for there is no point where there is complete discordance of phase for all kinds of light. In consequence, however, of the fact that the range of sensitiveness of the eye is limited to less than an "octave," the centre of the first dark band on either side is sensibly black ; but the existence of even one band is due to selection, and the formation of several visible bands is favoured by the capability of the retina to make chromatic distinctions within the range of vision. After two or three alternations the bands become highly coloured*; and, as the overlapping of the various elementary systems increases, the colours fado away, and the field of view assumes a uniform appearance.

There are, however, cases where it is possible to have systems of achromatic bands. For this purpose it is necessary, not merely that the maxima of illumination should coincide at some one place, but also that the widths of the bands should be the same for the various colours. The independence of colour, as we shall see, may be absolute; but it will probably be more convenient not to limit the use of the term so closely. The focal length of the ordinary achromatic object-glass is not entirely independent of colour. A similar use of the term would justify us in calling a system of bands achromatic, when the width of the elementary systems is a maximum or a minimum, for some ray very near the middle of the spectrum, or, which comes to the same, has equal values for two rays of finitely different refrangibility. The outstanding deviation from complete achromatism, according to the same analogy, may be called the secondary colour.

The existence of achromatic systems was known to Newton $\dagger$, and was insisted upon with special emphasis by Fox Talbot;; but singularly little attention appears to have been bestowed upon the subject in recent times. In the article "Wave Theory" (Encyc. Brit. 1888) I have discussed a few cases, but with too great brevity. It may be of interest to resume the consideration of these remarkable phenomena, and to detail some observations which I have made, in part since the publication of the 'Encyclopædia' article. A recent paper by M. Mascart $\S$ will also be referred to.

* The series of colours thus arising are calculated, and exhibited in the form of a curve upon the colour diagram, in a paper "On the Colours of Thin Plates," Edinb. Trans. 1887.

+ 'Opties,' Book ii.

$\ddagger$ Phil. Mag. [3] ix. p. 401 (1836).

"On the Achromatism of Interference," Comptes Rendus, March 1889; Phil. Mag. [5] xxvii. p. 519. 
Fresnel's Bands.

In this experiment the two sources of light which are regarded as interfering with one another must not be independent; otherwise there could be no fixed phase-relation. According to Fresnel's original arrangement the sources $O_{1}$, $\mathrm{O}_{2}$ are virtual images of a single source, obtained by reflexion in two mirrors. The mirrors may be replaced by a bi-prism. Or, as in Lloyd's form of the experiment, the second source may be obtained from the first by reflexion from a mirror placed at a high degree of obliquity. The screen upon which the bands are conceived to be thrown is parallel to $\mathrm{O}_{1} \mathrm{O}_{2}$, at distance $D$. If $A$ be the point of the sereen equidistant from $\mathrm{O}_{1}, \mathrm{O}_{2}$, and $\mathrm{P}$ a neighbouring point, then approximately $\mathrm{O}_{1} \mathrm{P}-\mathrm{O}_{2} \mathrm{P}=\sqrt{ }\left\{\mathrm{D}^{2}+\left(u+\frac{1}{2} b\right)^{2}\right\}-\sqrt{ }\left\{\mathrm{D}^{2}+\left(u-\frac{1}{2} b\right)^{2}\right\}=u b / \mathrm{D}$, where

$$
\mathrm{O}_{1} \mathrm{O}_{2}=b, \quad \mathrm{AP}=u \text {. }
$$

Thus, if $\lambda$ be the wave-length, the places where the phases are accordant are determined by

$$
u=n \lambda \mathrm{D} / b \text {, }
$$

$n$ being an integer representing the order of the band. The linear width of the bands (from bright to bright, or from dark to dark) is thus

$$
\Lambda=\lambda \mathrm{D} / b \text {. }
$$

The degree of homogeneity necessary for the approximate perfection of the $n$th band may be found at once from (1) and (2). For, if $d u$ be the change in $u$ corresponding to the change $d \lambda$, then

$$
d u / \Lambda=n d \lambda / \lambda
$$

Now clearly $d u$ must be a small fraction of $\Lambda$, so that $d \lambda / \lambda$ must be many times smaller than $1 / n$, if the darkest places are to be sensibly black. But the phenomenon will be tolerably well marked, if the proportional range of wave-length do not exceed $1 /(2 n)$, provided, that is, that the distribution of illumination over this range be not concentrated towards the extreme parts.

So far we have supposed the sources at $\mathrm{O}_{1}, \mathrm{O}_{2}$ to be mathematically small. In practice the source is an elongated slit, whose direction requires to be carefully adjusted to parallelism with the reflecting surface, or surfaces. By this means an important advantage is obtained in respect of brightness without loss of definition, as the various parts of the aperture give rise to coincident systems of bands.

The question of the admissible width of the slit requires

$$
\mathrm{H} 2
$$


careful consideration. We will suppose in the first place that the lights issuing from the various parts of the aperture are without permanent phase-relation, as when the slit is backed immediately by a flame, or by the incandescent carbon of an electric lamp. Regular interference can then only take place between lights coming from corresponding parts of the two images; and a distinction must be drawn between the two ways in which the images may be situated relatively to one another. In Fresnel's experiment, whether carried out with mirrors or with bi-prism, the corresponding parts of the images are on the same side; that is, the right of one corresponds to the right of the other, and the left of one to the left of the other. On the other hand, in Lloyd's arrangement the reflected image is reversed relatively to the original source: the two outer edges corresponding, as also the two inner. Thus, in the first arrangement the bands due to various parts of the slit differ merely by a lateral shift, and the condition of distinctness is simply that the width of the slit be a small proportion of the width of the bands. From this it follows as a corollary that the limiting width is independent of the order of the bands under examination. It is otherwise in Lloyd's method. In this case the centres of the systems of bands are the same, whatever part of the slit be supposed to be operative, and it is the distance apart of the images $(b)$ that varies. The bands corresponding to the various parts of the slit are thus upon different scales, and the resulting confusion inust increase with the order of the bands. From (1) the corresponding changes in $u$ and $b$ are given by

so that

$$
d u=-n \lambda \mathrm{D} d b / \dot{b}^{2} ;
$$

$$
d u / \Lambda=-n d b / b
$$

If $d b$ represents twice the width of the slit, (4) gives a measure of the resulting confusion in the bands. The important point is that the slit must be made narrower as $n$ increases, if the bands are to retain the same degree of distinctness.

If the various parts of the width of the slit do not act as independent sources of light, a different treatment would be required. To illustrate the extreme case, we may suppose that the waves issuing from the various elements of the width are all in the same phase, as if the ultimate source were a star situated a long distance behind. It would then be a matter of indifference whether the images of the slit, acting as proximate sources of interfering light, were reversed relatively to one auother, or not. It is, however, unnecessary to dwell upon this question, inasmuch as the conditions supposed are 
unfavourable to brightness, and therefore to be avoided in practice. The better to understand this, let us suppose that the slit is backed by the sun, and is so narrow that, in spite of the sun's angular magnitude, the luminous vibration is sensibly the same at all parts of the width. For this purpose the width must not exceed $\frac{1}{20}$ millim.* By hypothesis, the appearance presented to an eye close to the slit and looking backwards towards the sun will be the same as if the source of light were reduced to a point coincident with the sun's centre. The meaning of this is that, on account of the narrowness of the aperture, a point would appear dilated by diffraction until its apparent diameter became a large multiple of that of the sun. Now it is evident that in such a case the brightness may be enhanced by increasing the sun's apparent diameter, as can always be done by optical appliances. Or, which would probably be more convenient in practice, we may obtain an equivalent result by so designing the experiment that the slit does not require to be narrowed to the point at which the sun's image begins to be sensibly dilated by diffraction. The available brightness is then at its limit, and would be no greater, even were the solar diameter increased. The practical rule is that, when brightness is an object, slits backed by the sun should not be narrowed to much less than half a millimetre.

\section{Lloyd's Bands.}

Lloyd's experiment deserves to be more generally known, as it may be performed with great facility and without special apparatus. Sunlight is admitted horizontally into a darkened room through a slit situated in the window-shutter, and at a distance of 15 or 20 feet is received at nearly grazing incidence upon a vertical slab of plate glass. The length of the slab in the direction of the light should not be less than 2 or 3 inches, and for some special observations may advantageously be much increased. The bands are observed on a plane through the hinder vertical edge of the slab by means of a hand magnifying-glass of from 1 to 2 inch focus. The obliquity of the reflector is of course to be adjusted according to the fineness of the bands required.

From the manner of their formation it might appear that under no circumstances could more than half the system be visible. But, according to Airy's principle $f$, the bands may be displaced if examined through a prism. In practice all

* Verdet's Leçons d'Optique physique, t. i. p. 106.

+ See below. 
that is necessary is to hold the magnifyer somewhat excentrically. The bands may then be observed gradually to detach themselves from the mirror, until at last the complete system is seen, as in Fresnel's form of the experiment.

If we wish to observe interference under high relative retardation, we must either limit the light passing the first slit to be approximately homogeneous, or (aiter Fizeau and Foucault) transmit a narrow width of the band-system itself through a second slit, and subsequently analyse the light into a spectrum. In the latter arrangement, which is nsually the more convenient when the original light is white, the bands seen are of a rather artificial kind. If , apart from the heterogeneity of the light, the original bands are well formed, and if the second slit he narrow enough, the spectrum will be marked out into bands; the bright places corresponding to the kinds of light for which the original bands would be bright, and the black places to the kinds of light for which the original bands would be black. The condition limiting the width of the second slit is obviously that it be but a moderate fraction of the width of a band $(\Lambda)$.

If it be desired to pass along the entire series of bands up to those of a high order by merely traversing the second slit in a direction perpendicular to that of the light, a very long mirror is necessary. But when the second slit is in the region of the bands of highest order (that is, near the external limit of the field illuminated by both pencils), only the more distant part of the mirror is really operative; and thus, even though the mirror be small, bands of high order may be observed, if the second slit be carried backwards, keeping it of course all the time in the narrow doubly-illuminated field. In one experiment the distance from the first slit to the (3-inch) reflector was 27 feet, while the second slit was situated behind at a further distance of 4 feet. The distance $(b)$ between the first slit and its image in the reflector (measured at the window) was about 13 inches.

As regards the spectroscope it was found convenient to use an arrangement with detached parts. The slit and collimating lens were rigidly connected, and stood upon a long and rigid box, which carried also the mirror. The narrowness of the bands in which this slit is placed renders it imperative to avoid the slightest relative unsteadiness or vibration of these parts. The prisms, equivalent to about four of $60^{\circ}$, and the observing teleseope were upon another stand at a little distance behind the box which supported the rest of the apparatus.

Under these conditions it was easy to abserve bands in the spectrum whose width (from dark to dark) could be made as 
small as the interval between the $\mathrm{D}$ lines ; but for this purpose the first slit had to be rather narrow, and the direction of its length accurately adjusted, so as to give the greatest distinctness. Since the wave-lengths of the two D lines differ by about $T_{1000}^{1}$ part, spectral bands of this degree of closeness imply interference with a retardation of 1000 periods.

Much further than this it was not easy to go. When the bands were rather more than twice as close, the necessary narrowing of the slits began to entail a failing of the light, indicating that further progress would be attained with difficulty.

Indeed, the finiteness of the illumination behind the first slit imposes of necessity a somewhat sudden limit to the observable retardation. In this respect it is a matter of indifference at what angle the reflector be placed. If the angle be made small, so that the reflexion is very nearly grazing, the bands are upon a larger scale, and the width of the second slit may be increased, but in a proportional degree the width of the first slit must be reduced.

The relation of the width of the second slit to the angle of the mirror may be conveniently expressed in terms of the appearance presented to an eye placed close behind the former. The smallest angular distance which the slit, considered as an aperture, can resolve, is expressed by the ratio of the wave-length of light $(\lambda)$ to the width $\left(w_{2}\right)$ of the slit. Now, in order that this slit may perform its part tolerably well, $w_{2}$ must be less than $\frac{1}{2} \Lambda$; so that, by (2),

$$
\lambda / w_{2}>26 / \mathrm{D} \text {. . . . . . }
$$

The width must therefore be less than the half of that which would just allow the resolution of the two images (subtending the angle $b / D$ ) as seen by an eye behind. In setting up the apparatus this property may be turned to account as a test.

The existence of a limit to $n$, dependent upon the intrinsic brightness of the sun, may be placed in a clearer light by a rough estimate of the illumination in the resulting spectrum ; and such an estimate is the more interesting on account of the large part here played by diffraction. In most calculations of brightness it is tacitly assumed that the ordinary rules of geometrical optics are obeyed.

\section{Limit to Illumination.}

The narrowness of the second slit would not in itself be an obstacle to the attainment of full spectrum brightness, were we at liberty to make what arrangements we pleased behind 
it. In illustration of this, two extreme cases may be considered of a slit illuminated by ordinary sunshine. First, let the width $w_{2}$ be great enough not sensibly to dilate the solar image; that is, let $w_{2}$ be much greater than $\lambda / s$, where $s$ denotes in circular measure the sun's apparent diameter (about 30 minutes). In this case the light streams through the slit according to the ordinary law of shadows, and the pupil (of diameter $p$ ) will be filled with light if situated at a distance exceeding $d^{*}$, where

$$
p / d=s . \quad \text {. . . . . . }
$$

At this distance the apparent width of the slit is $w_{2} / d$, or $w_{2^{s}} / p$; and the question arises whether it lies above or below the ocular limit $\lambda / p$, that is, the smallest angular distance that can be resolved by an aperture $p$. The answer is in the affirmative, because we have already supposed that $w_{2} s$ exceeds $\lambda$. The slit has thus a visible width, and it is seen backed by undiffracted sunshine. If a spectrum be now formed by the use of dispersion sufficient to give a prescribed degree of purity, it is as bright as is possible with the sun as ultimate source, and would be no brighter even were the solar diameter increased, as it could in effect be by the use of a burningglass throwing a solar image upon the slit. The employment of a telescope in the formation of the spectrum gives no means of escape from this conclusion. The precise definition of the brightness of any part of the resulting spectrum would give opportunity for a good deal of discussion; but for the present purpose it may suffice to suppose that, if the spectrum is to be divided into $n$ distinguishable parts, so that its angular width is $n$ times the angular width of the slit, the apparent brightness is of order $1 / n$ as compared with that of the sun.

Under the conditions above supposed the angular width of the slit is in excess of the ocular limit, and the distance might be increased beyond $d$ without prejudice to the brilliancy of the spectrum. As the angular width decreases, so does the angular dispersion necessary to attain a given degree of purity. But this process must not be continued to the point where $w_{2} / d$ approaches the ocular limit. Beyond that limit it is evident that no accession of purity would attend an increase in $d$ under given dispersion. Accordingly the dispersion could not be reduced, if the purity is to be maintained; and the brightness necessarily suffers. It must always be a condition of full brightness that the angular width of the slit exceed the ocular limit.

Let us now suppose, on the other hand, that $w_{2}$ is so small

* About 30 inches. 
that the image of the sun is dilated to many times $s$, or that $w_{2}$ is much less than $\lambda / s$. The divergence of the light is now not $s$, but $\lambda / w_{2}$; and, if the pupil be just immersed,

$$
p / d=\lambda / w_{2} \text {. }
$$

The angular width of the slit $w_{2} / d$ is thus equal to $\lambda / p$, that is, it coincides with the ocular limit. The resulting spectrum necessarily falls short of full brightness, for it is evident that further brightness would attend an augmentation of the solar diameter, up to the point at which the dilatation due to diffraction is no longer a sensible fraction of the whole. In comparison with full brightness the actual brightness is of order $w_{2} s / \lambda$; or, if the purity required is represented by $n$, we may consider the brightness of the spectrum relatively to that of the sun to be of order $w_{2} s /(n \lambda)$.

In the application of these considerations to Lloyd's bands we have to regard the narrow slit $w_{2}$ as illuminated, not by the sun of diameter $s$, but by the much narrower source allowed by the first slit, whose angular width is $w_{1} / \mathrm{D}$. On this account the reduction of brightness is at least $w_{1} /(s \mathrm{D})$. If $v_{1}$ be so narrow as itself to dilate the solar image, a further reduction would ensue; but this could always be avoided, either by increase of $\mathrm{D}$, or by the use of a burning-glass focusing the sun upon the first slit. The brightuess of the spectrum of purity $n$ from the second slit is thus of order

$$
\frac{w_{1}}{s \mathrm{D}} \cdot \frac{w_{2} s}{n \lambda}=\frac{w_{1} w_{2}}{n \lambda \mathrm{D}}
$$

We have now to introduce the limitations upon $w_{1}$ and $w_{2}$. By (4) $w_{1}$ must not exceed $b /(4 n)$; and by (2) $w_{2}$ must not exceed $\lambda \mathrm{D} /(2 b)$. Hence the brightness is of order

$$
1 /\left(8 n^{2}\right), \text {. . . . . . }
$$

independent of $s$, and of the linear quantities. The fact that the brightness is inversely as the square of the number of bands to be rendered visible explains the somewhat sudden failure observed in experiment. If $n=2000$, the original brightness of the sun is reduced in the spectrum some 30 million times, beyond which point the illumination could hardly be expected to remain sufficient for vision of difficult objects such as narrow bands.

In Fresnel's arrangement, where the light is reflected perpendicularly from two slightly inclined mirrors, interference of high order is obtained by the movement of one of the mirrors parallel to its plane. The increase of $n$ does not then entail a narrowing of $w_{1}$; and bands of order $n$ may be 
observed in the spectrum of light transmitted through $w_{2}$, whose brightness is proportional to $n^{-1}$, instead of, as before, to $n^{-2}$.

\section{Achromatic Interference-Bands.}

We have already seen from (3) that in the ordinary arrangement, where the source is of white light entering through a narrow slit, the heterogeneity of the light forbids the visibility of more than a few bands. The scale of the various bandsystems is proportional to $\lambda$. But this condition of things, as we recognize from (2), depends upon the constancy of $l$, that is, upon the supposition that the various kinds of light all come from the same place. Now there is no reason why such a limitation should be imposed. If we regard $b$ as variable, we recognize that we have only to take $b$ proportional to $\lambda$, in order to render the band-interval $(\Lambda)$ independent of the colour. In such a case the system of bands is achromatic, and the heterogeneity of the light is no obstacle to the formation of visible bands of high order.

These requirements are very easily met by the use of Lloyd's mirror, and of a diffraction-grating, with which to form a spectrum. White light enters the dark room through a slit in the window-shutter, and falls in succession upon a grating, and upon an achromatic lens, so as to form a real diffraction-spectrum, or rather series of such, in the focal plane. The central image, and all the lateral coloured images except one, are intercepted by a screen. The spectrum wbich is allowed to pass is the proximate source of light in the interference experiment; and since the deviation of any colour from the central white image is proportional to $\lambda$, it is only necessary so to arrange the mirror that its plane passes through the white image in order to realize the conditions for the formation of achromatic bands.

There is no difficulty in carrying out the experiment practically. I have used the spectrum of the second order, as given by a photographed grating of 6000 lines in an inch, and a photographic portrait lens of about 6 inches focus. At a distance of about 7 feet from the spectrum the light fell upon a vertical slab of thick plate-glass 3 feet in length and a few inches high. The observer upon the further side of the slab examines the bands through a Coddington lens of somewhat high power, as they are formed upon the plane passing through the end of the slab. It is interesting to watch the appearance of the bands as dependent upon the degree in which the condition of achromatism is fulfilled. A comparatively rough adjustment of the slab in azimuth is sufficient 
to render achromatic, and therefore distinct, the first 20 or 30 bands. As the adjustment improves, a continually larger number become visible, until at last the whole of the doubly illuminated field is covered with fine lines.

In these experiments the light is white, or at least becomes coloured only towards the outer edge of the field. By means of a fine slit in the plane of the spectrum we may isolate any kind of light, and verify that the band-systems corresponding to various wave-lengths are truly superposed.

When the whole spectrum was allowed to pass, the white and black bands presented so much the appearance of a grating under the microscope that I was led to attempt to photograph them, with the view of thus forming a diffraction-grating. Gelatine plates are too coarse in their texture to be very suitable for this purpose; but I obtained impressions capable of giving spectra. Comparison with spectra from standard gratings showed that the lines were at the rate of 1200 to the inch. A width of about half an inch (corresponding to 600 lines) was covered, but the definition deteriorated in the outer half. A similar deterioration was evident on direct inspection of the bands, and was due to some imperfection in the conditions-perhaps to imperfect straightness of the slab. On one occasion the bands were seen to lose their sharpness towards the middle of the field, and to recover in the outer portion.

With respect to this construction of a grating by photography of interference-bands, a question may be raised as to whether we are not virtually copying the lines of the original grating used to form the spectrum. More may be said in favour of such a suggestion than may at first appear. For it would seem that the case would not be essentially altered if we replaced the real spectrum by a virtual one, abolishing the focusing lens, and bringing Lloyd's mirror into the neighbourhood of the grating. But then the mirror would be unnecessary, since the symmetrical spectrum upon the other side would answer the purpose as well as a reflexion of the first spectrum. Indeed, there is no escape from the conclusion that a grating capable of giving on the two sides similar spectra of any one order, without spectra of other orders or central image, must produce behind it, without other appliances and at all distances, a system of achromatic interference-fringes, which could not fail to impress themselves upon a sensitive photographic plate. But a grating so obtained would naturally be regarded as merely a copy of the first.

Another apparent anomaly may be noticed. It is found in 
practice that, to reproduce a grating by photography, it is necessary that the sensitive plate be brotight into close contact with the original ; whereas, according to the argument just advanced, no such limitation would be required.

These discrepancies will be explained if, starting from the general theory, we take into account the actual constitution of the gratings with which we can experiment. If plane waves of homogeneous light $(\lambda)$ impinge perpendicularly upon a plane $(z=0)$ grating, whose constitution is periodic with respect to $x$ in the interval $\sigma$, the waves behind have the general expression

$$
\begin{aligned}
\mathrm{A}_{0} \cos (k a t-k z) & +\mathrm{A}_{1} \cos \left(p x+f_{1}\right) \cos \left(k a t-\mu_{1} z\right) \\
& +\mathrm{B}_{1} \cos \left(p x+g_{1}\right) \sin \left(k a t-\mu_{1} z\right) \\
& +\mathrm{A}_{2} \cos \left(2 p x+f_{2}\right) \cos \left(k a t-\mu_{2} z\right)+\ldots ; .
\end{aligned}
$$

where

$$
p=2 \pi / \sigma, k=2 \pi / \lambda,
$$

and

$$
\mu_{1}^{2}=k^{2}-p^{2}, \quad \mu_{2}{ }^{2}=k^{2}-4 p^{2}, \quad \& c .,
$$

the series being continued as long as $\mu$ is real*. Features in the wave-form for which $\mu$ is imaginary are rapidly eliminated. For the present purpose we may limit our attention to the first three terms of the series, which represent the central image and the two lateral spectra of the first order.

When the first term occurs, as usually happens, the phenomena are complicated by the interaction of this term with the following ones, and the effect varies with $z$ in a manner dependent upon $\lambda$. This is the ordinary case of photographic reproduction, considered in the paper referred to. If $A_{0}$ vanish, there is no central image; but various cases may still be distinguished according to the mutual relations of the other constants. If only $\mathrm{A}_{1}$, or only $\mathrm{B}_{1}$, occur, we have interferencefringes. The intensity of light is (in the first case)

vanishing when

$$
\begin{aligned}
& \mathrm{A}_{1}^{2} \cos ^{2}(p x+f), \quad . \quad . \quad . \quad . \quad . \\
& p x+f=\frac{1}{2}(n+1) \pi ;
\end{aligned}
$$

and these fringes may be regarded as arising from the interference of the two lateral spectra of the first order,

$$
\begin{aligned}
& \frac{1}{2} \mathrm{~A}_{1} \cos \left(k a t-\mu_{1} z+p a+f_{1}\right), \\
& \frac{1}{2} \mathrm{~A}_{1} \cos \left(k a t-\mu_{1} z-p x-f_{1}\right) .
\end{aligned}
$$

As an example of only one spectrum, we may suppose

$$
\mathrm{B}_{1}=\mathrm{A}_{1}, \quad g_{1}=f_{1}-\frac{1}{2} \pi \text {, }
$$

* Phil. Mag. March 1881 ; Enc. Brit. Wave Theory, p. 440. 
giving

$$
\mathrm{A}_{1} \cos \left(k a t-\mu_{1} z-p x-f_{1}\right) . \quad . \quad . \quad .
$$

A photographic plate exposed to this would yield no impression, since the intensity is constant.

In order, then, that a grating may be capable of giving rise to the ideal system of interference-fringes, and thus impress itself upon a sensitive plate at any distance behind, the vibration due to it must be of the form

$$
\mathrm{A} \cos (p x+f) \cos \left(k a t-\mu_{1} z\right) . . \quad . \quad .
$$

It does not appear how any actual grating could effect this. Supposing $z=0$, we see that the amplitude of the vibration immediately behind the grating must be a harmonic function of $x$, while the phase is independent of $x$, except as regards the reversals implied in the variable sign of the amplitude. Gratings may act partly by opacity and partly by retardation, but the two effects would usually be connected; whereas the requirement here is that at two points the transmission shall be the same while the phase is reversed.

We can thus hardly regard the interference-bands obtained from a grating and Lloyd's mirror as a mere reproduction of the original ruling. As will be seen in the following paragraphs, much the same result may be got from a prism, in place of a grating; and if the light be sufficiently homogeneous to begin with, both these appliances may be dispensed with altogether.

\section{Prism instead of Grating.}

If we are content with a less perfect fulfilment of the achromatic condition, the diffraction-spectrum may be replaced by a prismatic one, so arranged that $d(\lambda / b)=0$ for the most luminous rays. The bands are then achromatic in the same sense that the ordinary telescope is so. In this case there is no objection to a merely virtual spectrum, and the experiment may be very simply executed with Lloyd's mirror and a prism of (say) $20^{\circ}$ held just in front of it.

The number of black and white bands to be observed is not so great as might perhaps have been expected. The lack of contrast which soon supervenes can only be due to imperfect superposition of the various component systems. That the fact is so is at once proved by observation according to the method of Fizeau ; for the spectrum from a slit at a very moderate distance out is seen to be traversed by bands. If the adjustment has been properly made, a certain region in the yellow-green is uninterrupted, while the closeness of the bands increases towards either end of the spectrum. So far 
as regards the red and blue rays, the original bands may be considered to be already obliterated, but so far as regards the central rays, to be still fairly defined. Under these circumstances it is remarkable that so little colour should be apparent on direct inspection of the bands. It would seem that the eye is but little sensitive to colours thns presented, perhaps on account of its own want of achromatism.

It is interesting to observe the effect of coloured glasses upon the distinctness of the bands. If the achromatism be in the green, a red or orange glass, so far from acting as an aid to distinctness, obliterates all the bands after the first few. On the other hand, a green glass, absorbing rays for which the bands are already confused, confers additional sharpness. With the aid of a red glass a large number of bands are seen distinctly, if the adjustment be made for this part of the spectrum.

A still better procedure is to isolate a limited part of the spectrum by interposed screens. For this purpose a real spectrum must be formed, as in the case of the grating above considered.

We will now inquire to what degree of approximation $\lambda / b$ may be made independent of $\lambda$ with the aid of a prism, taking Cauchy's law of dispersion as a basis. According to it the value of $b$ for any ray may be regarded as made up of two parts-one constant, and one varying inversely as $\lambda^{2}$. We therefore write

$$
\frac{\lambda}{b}=\frac{\lambda^{3}}{\mathrm{~A} \lambda^{2}-\mathrm{B}}, \quad \cdot \quad \cdot \quad \cdot \quad \cdot \quad \cdot
$$

where $\mathrm{A}$ is to be so chosen that $\lambda / b$ is stationary when $\lambda$ has a prescribed value, $\lambda_{0}$. This condition gives

so that

$$
\mathrm{A} \lambda_{0}{ }^{2}=3 \mathrm{~B} \text {; . . . . . . . (13) }
$$

$$
\frac{\lambda / b}{\lambda_{0} / b_{0}}=\frac{2}{3 \lambda_{0}} \frac{\lambda^{3}}{\lambda^{2}-\frac{1}{3} \lambda_{0}{ }^{2}} . \quad \text {. . . . . }
$$

As an example, let us suppose that the disposition is achromatic for the immediate neighbourhood of the line $\mathrm{D}$, so that $\lambda_{0}=\lambda_{\mathrm{D}}$, and inquire into the proportional variation of $\lambda / b$, when we consider the ray C. Assuming

we obtain from (14)

$$
\lambda_{\mathrm{D}}=\cdot 58890, \quad \lambda_{\mathrm{c}}=\cdot 65618,
$$

$$
\frac{\lambda / b}{\lambda_{0} / b_{0}}=1 \cdot 0155 \text {. }
$$

The meaning of this result will be best understood if we inquire for what order $(n)$ the bands of the C-system are 
shifted relatively to those of the D-system through half the band-interval. From (1)

by hypothesis ; so that

$$
\begin{aligned}
\delta u & =n \mathrm{D}\left\{\lambda / b-\lambda_{0} / b_{0}\right\} \\
& =\frac{1}{2} \lambda_{0} \mathrm{D} / b_{0}
\end{aligned}
$$

$$
n=\frac{\frac{1}{2} \lambda_{0} / b_{0}}{\lambda / b-\lambda_{0} / b_{0}} . \quad . \quad . \quad . \quad . .
$$

Thus, in the case supposed, $n=32$. After 32 periods the black places of the C-system will coincide with the bright places of the D-system, and conversely. If no prism had been employed ( $b$ constant), a similar condition of things would have arisen when

$$
n=\frac{\frac{1}{2} \lambda_{0}}{\lambda-\lambda_{0}}=4 \cdot 2 .
$$

If $\left(\lambda-\lambda_{0}\right)$ or, as we may call it, $\delta \lambda$ be small,

$$
\frac{\lambda / b-\lambda_{0} / b_{0}}{\lambda_{0} / b_{0}}
$$

is of the second order in $\delta \lambda$. An analytical expression is readily obtained from (14). We have

$$
\begin{aligned}
\frac{\lambda / b}{\lambda_{0} / b_{0}} & =\frac{1+3 \delta \lambda / \lambda_{0}+3\left(\delta \lambda / \lambda_{0}\right)^{2}+\left(\delta \lambda / \lambda_{0}\right)^{3}}{1+3 \delta \lambda / \lambda_{0}+\frac{3}{2}\left(\delta \lambda / \lambda_{0}\right)^{2}} \\
& =1+\frac{\frac{3}{2}\left(\delta \lambda / \lambda_{0}\right)^{2}+\left(\delta \lambda / \lambda_{0}\right)^{3}}{1+3 \delta \lambda / \lambda+\frac{3}{2}\left(\delta \lambda / \lambda_{0}\right)^{2}} \\
& =1+\frac{3}{2}\left(\delta \lambda / \lambda_{0}\right)^{2}-\frac{7}{2}\left(\delta \lambda / \lambda_{0}\right)^{3},
\end{aligned}
$$

approximately ; so that, by (15),

$$
n=\frac{1}{3}\left(\frac{\lambda_{0}}{\delta \lambda}\right)^{2}\left\{1+\frac{7}{3} \frac{\delta \lambda}{\lambda_{0}}+\ldots\right\} . . .
$$

This gives the order of the band at which complete discrepance first occurs for $\lambda_{0}$ and $\lambda_{0}+\delta \lambda$, the adjustment being made for $\lambda_{0}$. It is, of course, inversely proportional to the square of $\delta \lambda$, when $\delta \lambda$ is small.

The corresponding value of $n$, if no prism be used, so that $b$ is constant, is

$$
n=\frac{1}{2} \frac{\delta \lambda}{\lambda_{0}} . \quad \text {. . . . . . }
$$

The effect of the prism is thus to increase the number of bands in the ratio

$$
2 \lambda_{0}: 3 \delta \lambda \text {. }
$$

[To be continued.] 